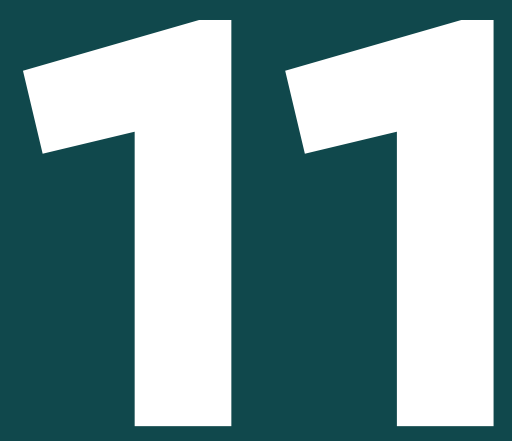

\title{
La Pobreza como Violación de los Derechos Humanos: El Resultado de un Sistema Económico Desigual
}

Poverty as a Violation of Human Rights: The Result of a Globally Unequal Economic and Political System

FECHA DE RECEPCIÓN: 27/07/2020

FECHA DE APROBACIÓN: 11/03/2021 


\section{Resumen}

\section{Paul Sebastián León Gómez¹ |}

Las instituciones intergubernamentales emitieron declaraciones y tratados como un estándar común de logros para todos los pueblos y naciones, con el objetivo de proteger los derechos humanos, incluido el derecho a la salud. Sin embargo, la pobreza, que constituye el determinante social más importante de la salud, no ha sido reconocida como una violación de los derechos humanos. Este artículo argumentó que la pobreza y, por ende, la mala salud, surge de las desigualdades sociales y económicas, constituyendo una violación directa de los derechos humanos. Para ello, se examinaron los principales tratados de derechos humanos, como la Declaración Universal de Derechos Humanos (DUDH) y el Pacto Internacional de Derechos Económicos, Sociales y Culturales (ICESCR, por sus siglas en inglés) mientras se integró la bibliografía y estadísticas disponibles de artículos científicos y páginas web oficiales. La investigación mostró que las personas con escasos recursos económicos tienen menos probabilidades de acceder a servicios de salud y de participar en actividades educativas. También, el estudio indicó que uno de los principales factores que contribuyen a la pobreza son las reformas económicas impulsadas por multimillonarios y organizaciones supranacionales, las cuales, obstaculizan la promoción del bienestar común. Además, las decisiones políticas adoptadas por los países industrializados para promover conflictos armados en los países en vías de desarrollo, han hecho que la pobreza se arraigue aún más. Se concluyó que es necesario introducir reformas económicas y políticas que alivien la pobreza y disminuyan las desigualdades de ingresos, aún cuando estas vayan en contra del sistema económico.

Palabras clave: derecho a la salud, derechos humanos, desigualdad económica, instituciones intergubernamentales, pobreza,

Naciones Unidas.

1 Universidad del Azuay ·psleon@uazuay.edu.ec • https://orcid.org/0000-0001-9094-490X • Cuenca, Ecuador 


\section{Abstract}

I

ntergovernmental institutions issued declarations and treaties as a common standard of achievements for all peoples and all nations. Those charters are aimed to protect human rights including the right to health. Yet, poverty, which constitutes the most important social determinant of health, is not recognized as a violation of human rights. This paper argues that poverty and, thereby, poor health emerge from income and social inequalities, which constitutes a direct violation of human rights. To that end, the study discusses major human rights declarations and treaties such as the Universal Declaration of Human Rights (UDHR), and the International Covenant on Economic, Social and Cultural Rights (ICESCR) while integrating the available literature and statistics from scientific articles and official webpages. This paper shows that poor individuals are less likely to access health services and to participate in educational, and recreational activities which constitute a direct threat towards the individual's health. It also claims that a major contributor to poverty is the economic reforms lobbied by billionaires and supranational organizations which hinders the promotion and maintenance of the common well-being. In addition, the political decisions taken by industrialized countries to promote armed conflicts in developing states have caused poverty to develop even deeper roots in today's international order. Therefore, there is a need for major economic and political reforms that relieves poverty and decreases inequality, even if it goes against the current economic system.

Keywords: economic inequality, human rights, intergovernmental institutions, poverty, right to health, United Nations. 


\section{Introducción}

V

arias instituciones intergubernamentales como las Organización de la Naciones Unidas (ONU), han emitido declaraciones y tratados con el fin de proteger los derechos humanos. Dos de los más importantes han sido la Declaración Universal de Derechos Humanos (1948) y el Pacto Internacional de Derechos Económicos, Sociales y Culturales (1966), que reconocen a la salud como un derecho humano fundamental, sin establecer la pobreza como una violación de los derechos humanos, a pesar de ser el determinante social más importante de la salud. En las últimas décadas, se ha reconocido cada vez más a la pobreza como un problema de derechos humanos. Los defensores de derechos humanos han empezado a tomarse en serio los derechos económicos, sociales y culturales y a reconocer la centralidad de la pobreza en muchas violaciones de derechos humanos. En el seno de la Organización de las Naciones Unidas (ONU), después de la Conferencia Mundial de Derechos Humanos celebrada en Viena en 1993, se afirmó la indivisibilidad, interdependencia e interrelación de todos los derechos humanos (Costa, 2008, p.81).

Sin embargo, estas afirmaciones muy amplias no ayudan a clarificar el complejo problema de clasificar la pobreza como una violación de los derechos humanos. Los principales intentos en este sentido se llevaron a cabo en la ONU, por parte del Programa de las Naciones Unidas para el Desarrollo (PNUD) y la Oficina del Alto Comisionado para los Derechos Humanos (OACDH) (Costa, 2008, p.82). Casi todos estos esfuerzos se realizaron en el marco de las reformas introducidas por el Secretario General en 1997, referente al entendimiento común de las Naciones Unidas sobre el enfoque del desarrollo basado en los derechos humanos (Human Rights Approach, 2003, p.5). 
Por lo tanto, estas declaraciones de la ONU se dirigen, principalmente, a los funcionarios encargados de la reducción de la pobreza, explicándoles cómo debe aplicarse el enfoque de la integración de los derechos humanos a su trabajo en la vida real. Sin embargo, los expertos internacionales en derechos humanos siguen sin tener claro el significado exacto de la afirmación de que la pobreza viola los derechos humanos (Costa, 2008, p.82). Esto sucede porque no se ha desarrollado un marco conceptual que vincule los determinantes de la pobreza con los determinantes de la violación de derechos humanos específicos (por ejemplo, el derecho a la salud, la educación, alimentación), para mostrar cómo se refuerzan mutuamente (Schuftan, 2012, p.486).

Este estudio trata de aclarar este vacío conceptual, presentando un resumen crítico de la conexión entre la pobreza y los derechos humanos analizando los diferentes marcos conceptuales, los principales tratados y acuerdos sobre los derechos humanos y la literatura académica. En la primera sección se abordan las definiciones de pobreza y derechos humanos, como primer paso para construir una claridad conceptual. Luego, el estudio compila datos estadísticos de investigaciones y sitios oficiales para explicar la pobreza como una violación de los derechos humanos (Costa, 2008, p.83). Además, se procede a una discusión sobre cómo surge la pobreza, resultado de las desigualdades sociales y económicas, las cuales, han sido perpetuadas a través de reformas económicas y políticas impulsadas por empresas multinacionales y organizaciones supranacionales.

Para entender por qué la pobreza es una violación directa de los derechos humanos, es necesario definir qué se entiende por derechos humanos. Según la ONU, "los derechos humanos son derechos inherentes a todos los seres humanos, sin distinción alguna de raza, sexo, nacionalidad, origen étnico, lengua, religión o cualquier otra condición". Algunos de los derechos humanos más significativos incluyen el derecho a la vida, libertad de expresión, educación, trabajo y salud (What are your human rights?, s.f). La DUDH (1948) sostiene que los seres humanos tienen derecho a vivir con dignidad e igualdad. Varios países firmaron tratados internacionales que les proporcionan un conjunto de directrices destinadas a garantizar los derechos humanos (What are your human rights?, s.f).

A pesar de que se han creado declaraciones y tratados para promover y proteger los derechos humanos, haciendo hincapié en el dere- 
cho a la salud, se ha excluido a la pobreza como una violación de dichos derechos. La DUDH (1948) establece que los seres humanos tienen derecho a disfrutar de todos los derechos mencionados, independientemente de la raza, el sexo, la religión, la propiedad y el origen social. Esto sugiere que la declaración no tiene en cuenta los problemas que enfrentan la población económicamente desfavorecida en materia de derechos humanos. En cuanto a la salud, la DUDH (1948) menciona que «toda persona tiene derecho a un nivel de vida adecuado que le asegure, así como a su familia, la salud y el bienestar». De manera similar, el ICESCR (1966) y la Organización Mundial de la Salud (OMS) señalan que todos los seres humanos tienen derecho a disfrutar del «más alto nivel posible de salud» (Constitution of the WHO, 1967, p. 1). Esta declaración sugiere que el derecho a la salud no significa necesariamente que las personas tengan el derecho a estar sanas; más bien, significa que la salud de las personas depende de los recursos de salud que el Estado les proporciona. Además, la declaración del ICESCR (1966) considera que algunos de los determinantes sociales de la salud, tales como el saneamiento adecuado, la vivienda y la educación son esenciales para lograr un nivel de salud óptimo. Así pues, los estados signatarios están obligados no solo a prestar servicios de salud de buena calidad, sino también, abordar esos factores subyacentes. Benatar (1988, p. 296) afirma que aquellos tratados internacionales tienen por objeto fomentar el derecho internacional y el respeto a la dignidad humana, rechazando la explotación que sufren ciertos sectores de la sociedad. A pesar de ello se centran, principalmente, en discutir la importancia de garantizar los servicios de salud adecuados, sin reconocer los efectos negativos que tiene la pobreza.

Para examinar más a fondo la manera en que la pobreza viola los derechos humanos, es importante definir qué se entiende por pobreza y cómo se delimita. Cuando los ingresos de un individuo no alcanzan un límite económico esencial para pagar servicios humanos básicos se denomina spobreza absolutas. Por ejemplo, en 2015, el Banco Mundial estableció que el umbral internacional de pobreza es de $\$ 1,90$ dólares por día (FAQs: Global Poverty Line, 2015). Sin embargo, esta definición no considera la desigualdad y calidad de vida. Por lo tanto, la pobreza también puede definirse en relación con la situación económica de otras personas en un concepto conocido como pobreza relativa. Este término se refiere a cuando una persona no cumple con los estándares de condiciones de vida establecidos en relación con el entorno socioeconómico 
en el que vive (Migration and inclusive societies, s.f). Por ejemplo, en 2018, en Ecuador, la línea de pobreza se ubicó en US\$ 84,72 mensuales por persona (Coordinación General de Innovación en Métricas y Análisis de la Información, 2018, p.2). Este artículo usa la definición de pobreza relativa.

En el debate sobre el derecho a la salud, los determinantes sociales desempeñan un papel crucial ya que un entorno de vida inadecuado, combinado con un comportamiento insalubre, es una amenaza directa para la salud (Social Determinants of Health: Solid Facts, 2003). La insalubridad se refiere, entre otros, al acceso limitado a los alimentos, inestabilidad residencial y el acceso inadecuado al agua y a otros servicios de saneamiento (Social Determinants of Health, s.f). Algunos de los comportamientos perjudiciales para la salud incluyen la falta de actividad física, el consumo de tabaco, alcohol y azúcar (Mikkonen y Raphael, 2010, p. 7). Los determinantes sociales están fuertemente conectados entre sí. Por ejemplo, el acceso a la educación reduce las posibilidades de tener conductas poco saludables $y$, al mismo tiempo, las personas con niveles de educación más altos, tienden a tener un mejor acceso a la alimentación y vivienda (Social Determinants of Health, s.f). La tabla 1 muestra el porcentaje de acceso a distintos tipos de vivienda por nivel educativo. Los datos muestran que los encuestados que viven en viviendas privadas, tienen un nivel educativo más alto (67\%); mientras que, la gran mayoría (61\%) de personas que tienen un título de bachiller o no finalizaron el colegio, tan solo pueden acceder a viviendas públicas de alquiler (Tabla 1). Para la recogida de datos, este estudio realizó una encuesta de hogares con la ayuda de una empresa inmobiliaria de Hong Kong. La estrategia consistía en abarcar los principales sectores de la vivienda. La parte que corresponde al eje ' $x$ ' representa los 3 niveles de educación considerados: escuela, colegio y universidad. El eje ' $y$ ' muestra los diferentes tipos de vivienda: privadas y de arriendo (Gou; et al, 2018). 
Tabla 1. Sector de la vivienda por nivel educativo

\begin{tabular}{ccccc}
\hline & \multicolumn{4}{c}{ Education $\left(\mathrm{x}^{2}=\mathbf{0 . 0 0 0}\right)$} \\
\cline { 2 - 5 } Housing Types & $\begin{array}{c}\text { Primary } \\
\text { Education }\end{array}$ & $\begin{array}{c}\text { Secondary } \\
\text { Education }\end{array}$ & $\begin{array}{c}\text { Tertiary and } \\
\text { Higher Education }\end{array}$ & Total \\
\hline Public Rental Housing & $16 \%$ & $45 \%$ & $39 \%$ & $100 \%$ \\
Public Rental Housing & $4 \%$ & $51 \%$ & $45 \%$ & $100 \%$ \\
Private Housing & $4 \%$ & $29 \%$ & $67 \%$ & $100 \%$ \\
\hline
\end{tabular}

Tabla 1. (Gou, Z., Xie, X., Lu, Y \& Khoshbakht, M. (2018). Quality of Life (QoL) Survey in Hong Kong: Understanding the Importance of Housing Environment and Needs of Residents from Different Housing Sectors. International Journal of Environmental Research and Public Health, 15, 219. Doi:10.3390/ijerph15020219

Otro de los principales determinantes de la salud es la distribución de la riqueza. En esto influyen mucho las decisiones de los gobiernos en el plano local, nacional e internacional, porque determinan la cantidad de ingresos que recibe la población por concepto de servicios sociales, empleo y prestaciones gubernamentales (Mikkonen y Raphael, 2010, p. 7-8). Por lo tanto, una distribución desigual de los ingresos conduce a la pobreza, lo que a su vez dificulta el acceso a otros determinantes sociales de la salud, como la alimentación, servicios de salud, educación y vestimenta. 


\section{Metodología}

Este estudio sintetiza la literatura disponible examinando los principales tratados de derechos humanos, tales como la Declaración Universal de Derechos Humanos (DUDH) y el Pacto Internacional de Derechos Económicos, Sociales y Culturales (ICESCR, por sus siglas en inglés) integrando, al mismo tiempo, la bibliografía disponible. Para la selección de artículos se realizaron búsquedas en PubMed and Web of Science (incluyendo Web of Science ${ }^{\mathrm{TM}}$ Core Collection, BIOSIS previews y SciELO Citation Index, cuyo contenido incluye revistas regionales de América Latina y Norteamérica, así como títulos de España y Hong Kong) (Liu; et al, 2014, p. 3) utilizando palabras clave variables para identificar artículos con resúmenes publicados entre enero de 1995 y diciembre 2020, centrados en la discusión de la salud como un derecho humano. Se evaluaron el contenido de los artículos que cumplían los criterios de inclusión para garantizar su relevancia. Se consideraron todos los artículos independientemente del enfoque metodológico, incluyendo metodologías cualitativas y/o cuantitativas (Bills and James, 2016, p. 688). Este estudio también integra artículos de periodismo y páginas web oficiales, los cuales, debían cumplir los siguientes criterios de inclusión: Idioma inglés o español, inclusión del nombre de autores, fecha de publicación y la descripción del sitio web incluyendo misión y valores. Los términos de búsqueda clave incluían iteraciones variables de las siguientes palabras clave: derechos humanos, pobreza, derecho a la salud y desigualdad económica, ONU. 


\section{Resultados}

La pobreza es el determinante social más importante de la salud, porque las personas con falta de recursos económicos se enfrentan a privaciones sociales y materiales como, por ejemplo, una mayor dificultad para acceder a un seguro de salud. El gráfico 1 muestra la población ecuatoriana no afiliada al seguro. El eje ' $x$ ' se refiere a la edad de las personas; mientras que, el eje ' $y$ ' muestra la prevalencia en términos de porcentaje de la población ecuatoriana no afiliada al seguro de salud (Llerena, Pinto and Pinto, 2017, p.3). La proporción de población no afiliada a un seguro de salud asciende al $81 \%$ en los hogares del Quintil 1; mientras que, la proporción no cubierta por un seguro de vida del Quintil 4 y 5 es del $28 \%$. Esta figura indica claramente que la cobertura de seguro de salud depende de la distribución de ingresos; a menor ingreso económico menor acceso a un seguro de salud (Gráfico 1). Dichos datos fueron obtenidos a través de la Encuesta Nacional de Empleo, Desempleo y Subempleo-ENEMDU en 2016 (Llerena, Pinto y Pinto, 2017, p. 2-4).

Gráfico 1. Población no cubierta o afiliada por Seguro de Salud

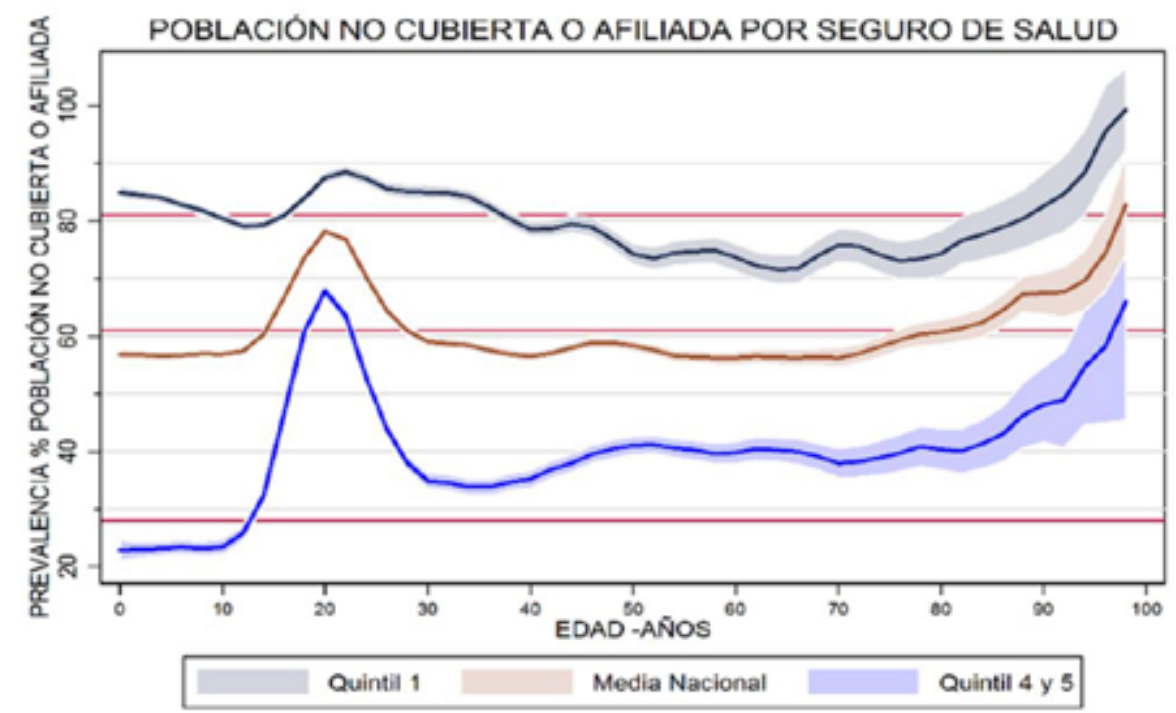

Fuente: Foro Economía Ecuador. (Llerena, F., Pinto, M, A., \& Pinto, M, C., 2017). 
Además, los niveles de ingresos tienen un papel más importante en países que ofrecen un acceso limitado a servicios sanitarios y sociales como Ecuador y, por consiguiente, las familias con falta de recursos tienden a sufrir de niveles más bajos de salud (Mikkonen y Raphael, 2010, p. 12). Por ejemplo, la mayoría de muertes evitables se producen en las naciones del tercer mundo, principalmente, entre los sectores más pobres (Phillips, 2011, p. 406). En las últimas siete décadas, el hambre y la malnutrición, que surgieron como resultado de la pobreza, han matado a más personas que todos los conflictos armados juntos (Russell, 1998, p. 354).

De igual manera, la pandemia del Covid-19 afectó desproporcionalmente a los individuos con mayores tasas de pobreza, ya que estos son más propensos a morir al contagiarse del virus (Chen, Waterman, Krieger, 2020, p. 1). El gráfico 2 indica las diferencias en la tasa de mortalidad por nivel de ingresos en la ciudad de Massachusetts, USA, antes y durante de la pandemia del Covid-19. El gráfico se divide en dos recuadros; el primero, muestra la tasa de mortalidad en el año 2020; y, el segundo recuadro, muestra la tasa de mortalidad durante los años 2015-2019. El eje ' $x$ ' se refiere a las fechas cuando se recogieron los datos; mientras que, el eje ' $y$ ' muestra la diferencia en la tasa de mortalidad por cada 100 mil personas. La línea morada se refiere a los hogares con mayores niveles de pobreza; la línea verde representa a los hogares con un nivel de pobreza menor al grupo morado, pero, mayor a los grupos amarillo y azul; y, la línea azul se refiere a las familias con el mejor nivel socioeconómico de los cuatro grupos (Chen, Waterman, Krieger, 2020, p. 1-3). Las estadísticas señalan que cuando la pandemia explosionó en USA y la mortalidad se disparó, los hogares más pobres fueron los que más sufrieron un incremento en el número de muertes (gráfico 2). Dichos datos fueron obtenidos a partir de los reportes de mortalidad que maneja el municipio de Massachusetts durante los últimos cinco años (Chen, Waterman, Krieger, 2020, p. 2-3). 
Gráfico 2. Esta figura indica las diferencias en la tasa de mortalidad por nivel de ingresos en la ciudad de Massachusetts, USA.

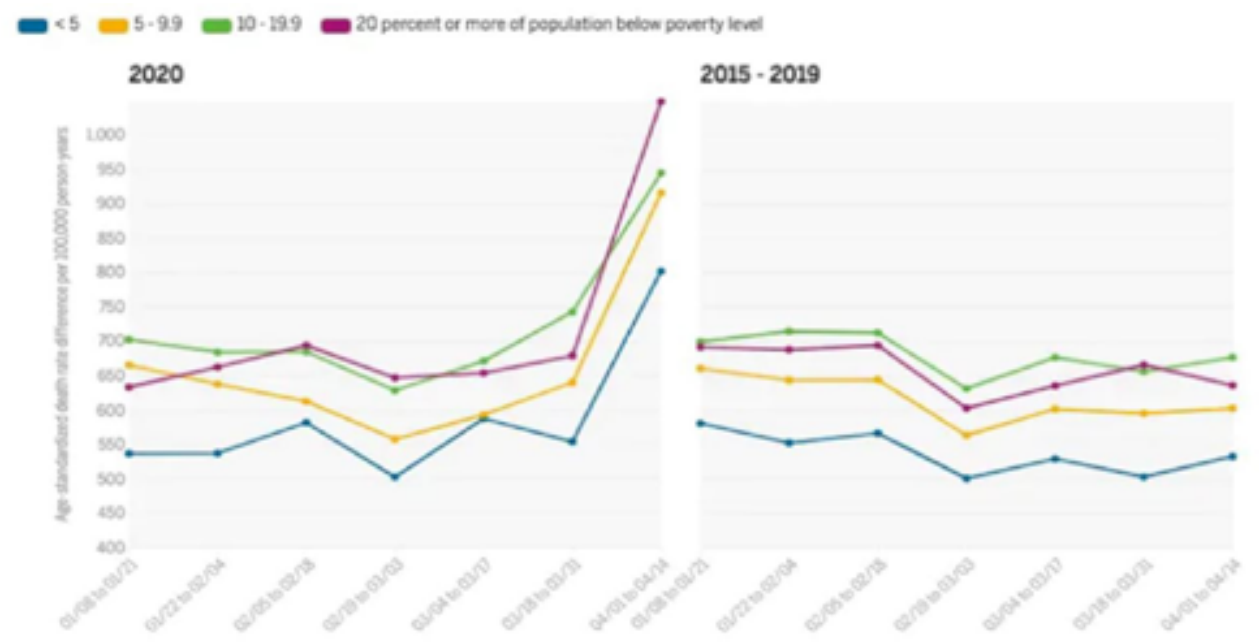

Fuente: Harvard Center for Population and Development Studies (Chen, J., Waterman, D., \& Krieger N., 2020).

La pobreza y, por consiguiente, la mala salud, surgen de las desigualdades de ingresos, lo que constituye una violación directa a los derechos humanos. El sistema creado por las élites económicas y políticas, perpetúa la excesiva concentración de la riqueza y obstaculiza la promoción y el mantenimiento del bienestar común (Schuftan, 2012, p. 487). Esta injusta distribución de los ingresos se produce a nivel local, nacional e internacional (Commission on Social Determinants of Health, 2008). La DUDH (1948), en su primer artículo, establece que «todos los seres humanos nacen libres e iguales en dignidad y derechos»; sin embargo, esto no ocurre. Por ejemplo, no hay igualdad entre un niño nacido en un barrio rico de un país industrializado y un niño nacido en un suburbio de bajos ingresos en Latinoamérica. A pesar de que el niño tiene derechos humanos, en la práctica, tal vez no disfrute a plenitud de ellos, tales como el acceso a una alimentación sana, a una vivienda digna o a la educación (Benatar, 1988, p. 298). Otro artículo de la DUDH (1948) declara que toda persona tiene derecho a un nivel de vida adecuado para su salud. Lamentablemente, las estadísticas muestran que los países 
más desiguales suelen tener peores resultados en materia de salud. El gráfico 3 indica el índice de problemas sanitarios y sociales en relación con la desigualdad de ingresos en varios países. El índice combina datos sobre: esperanza de vida, logros mentales, desconfianza y movilidad social. El eje ' $x$ ' se refiere a la desigualdad de ingresos, siendo la parte derecha del gráfico la zona con más inequidad. Mientras que el eje ' $y$ ', indica el índice de problemas sociales y de salud, siendo la parte superior la zona que más problemas sociales y de salud tiene. La línea en el medio indica la correlación entre las dos variables. Se puede observar una clara correlación: entre a mayor desigualdad, mayor número de problemas de salud. En este caso, USA y Portugal, representan los países con una mayor inequidad salarial, lo cual, se traduce a un mayor número de problemas de salud entre sus ciudadanos (Gráfico 3) (Pickett y Wilkinson, 2015, p. 315).

Gráfico 3. Income inequality and health: A causal review.

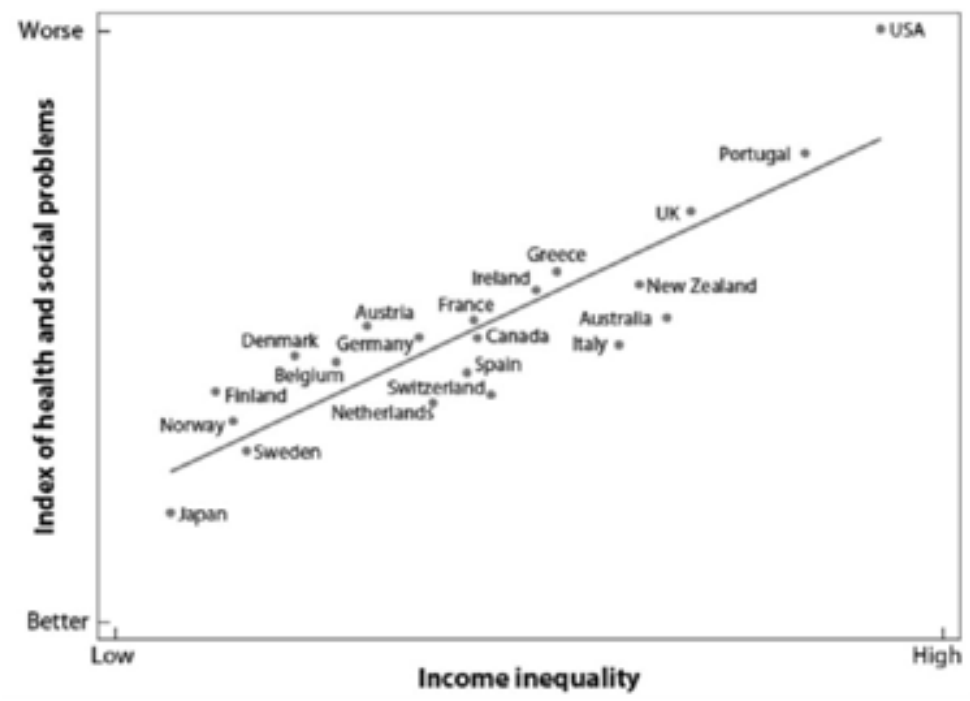

Fuente: Social Science \& Medicine (Pickett, K \& Wilkinson, R., 2015). 
Otro ejemplo de esto es que después de la Segunda Guerra Mundial, mientras los Estados Unidos ocupaba el primer lugar en la tabla de esperanza de vida mundial, Japón, una sociedad más desigual en ese momento, ocupaba un lugar significativamente inferior; no obstante, durante la década de los 80 , la desigualdad en los EE.UU. aumentó significativamente $y$, en consecuencia, su posición en la tabla de esperanza de vida cayó algunas posiciones. Por otro lado, Japón hizo importantes esfuerzos para reducir la brecha de la desigualdad, lo que llevó al país asiático a la posición número uno en esperanza de vida en el mundo (Pickett y Wilkinson, 2015, p. 317).

Además, estudios afirman que las tasas de violencia, que afectan directamente a la salud de las personas, aumentan considerablemente en las regiones donde la desigualdad de ingresos tiende a ser mayor (The stark relationship between income, 2018). El gráfico 4 muestra la correlación entre las percepciones de crimen y la desigualdad de ingresos de personas en 142 países. El eje ' $x$ ' indica el nivel de desigualdad económica; el eje ' $y$ ' en el recuadro de la izquierda se refiere, en términos de porcentaje a propiedades o dinero robado; mientras que, el recuadro de la derecha presenta en porcentaje los asaltos que sufrieron las personas entrevistadas. Cada país es representado con un punto. Para obtener estas estadísticas, la organización de sondeos Gallup preguntó a 148 mil personas de 142 países, sobre su percepción de la delincuencia y su grado de seguridad en dos aspectos: si les han robado bienes o dinero y si han sido agredidos en el último año. Los datos indican que existe una correlación fuerte y positiva entre estas percepciones de crimen y la cantidad de desigualdad de ingresos (gráfico 4) ("The stark relationship between income", 2018). La desigualdad sigue aumentando en la mayoría de los países porque ni las organizaciones ni los dirigentes políticos se centran en la reducción de la brecha de ingresos (Schuftan, 2012, p. 490). Por lo tanto, la protección de los derechos humanos se ve amenazada cuando los asuntos económicos y políticos se convierten en el tema de debate más importante para los gobiernos (Benatar, 1988, p. 298). 
Gráfico 4. The stark relationship between income inequality and crime.

Have had money or property stolen, \%

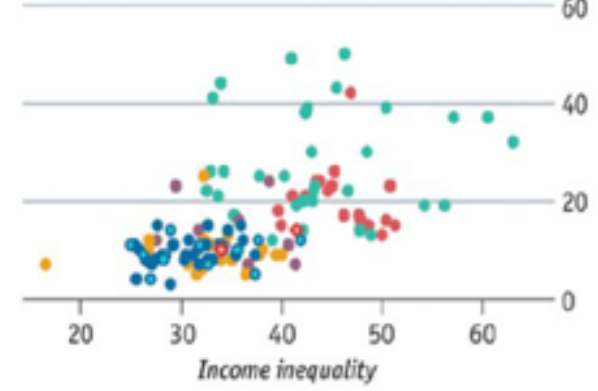

Sources: Gallup; World Bank; The Economist Economist.com
Have been assaulted, $\%$
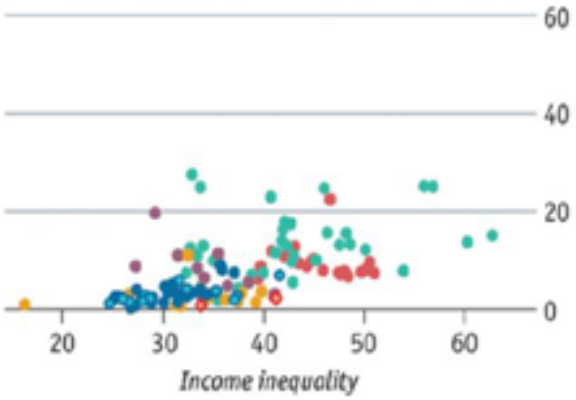

-Latest arailable, 0 =perfect equality and 100 - perfect inecuality

Fuente: Keeping up with the Joneses. The Economist. (2018). 


\section{Discusión}

Uno de los principales contribuyentes a la pobreza y, por consiguiente, a la violación del derecho a la salud son las reformas impulsadas por el sistema económico actual y las instituciones supranacionales y privadas. El sistema económico actual condujo a la división de la población en una pequeña clase rica, ubicada principalmente en países occidentales y, los pobres, que constituyen la mayoría de la población. Las élites tienen una enorme influencia en el proceso de toma de decisiones políticas; por lo tanto, apoyan ciertas políticas económicas que favorecen la extracción de bienes humanos y materiales de los países en desarrollo (Benatar, 1988, p. 296). Los fundamentalistas del mercado pasan por alto los principios básicos de la salud pública porque la salud, en lugar de ser un derecho, se convierte en una comodidad que no todo el mundo puede acceder. Además, este sistema económico da prioridad al Producto Nacional Bruto (PNB) sobre los derechos humanos, incluido el derecho a la salud, lo cual, lleva a que la salud se convierta en una herramienta necesaria para la productividad. Por lo tanto, grandes porciones de la sociedad que no contribuyen a aumentar el PNB, como los discapacitados y presos (gráfico 5), tienen dificultades para acceder a los servicios de salud (Katz, 2008, p. 1-2). El gráfico 5 muestra la distribución de las causas de muerte, según categorías entre presos. Este estudio documentó los fallecimientos en una prisión española entre 1994 y 2004. Se recogieron las variables edad, sexo, fecha del óbito, serología VIH y causa de muerte, según la clasificación: muerte por $\mathrm{VIH}$, por enfermedad no $\mathrm{VIH}$, suicidio, intoxicación por drogas, y accidentes. Los autores de este proyecto establecieron la tendencia de las tasas de mortalidad mediante un modelo de regresión lineal. Respecto a las causas de fallecimiento el $45 \%$ de internos fallecieron por causas directamente relacionadas con el VIH, 7\% por suicidio y 7\% por intoxicación por drogas (gráfico 5) (Vera, Planelles, García, 2005, p. 678). Es decir, alrededor del $60 \%$ de fallecidos en esta prisión fue producto de muertes prevenibles, relacionados con falta de servicios públicos en salud sexual y mental, demostrando un total abandono por parte de las instituticiones estatales y locales. 
Gráfico 5. Tendencia de la tasa de mortalidad en una prisión española.

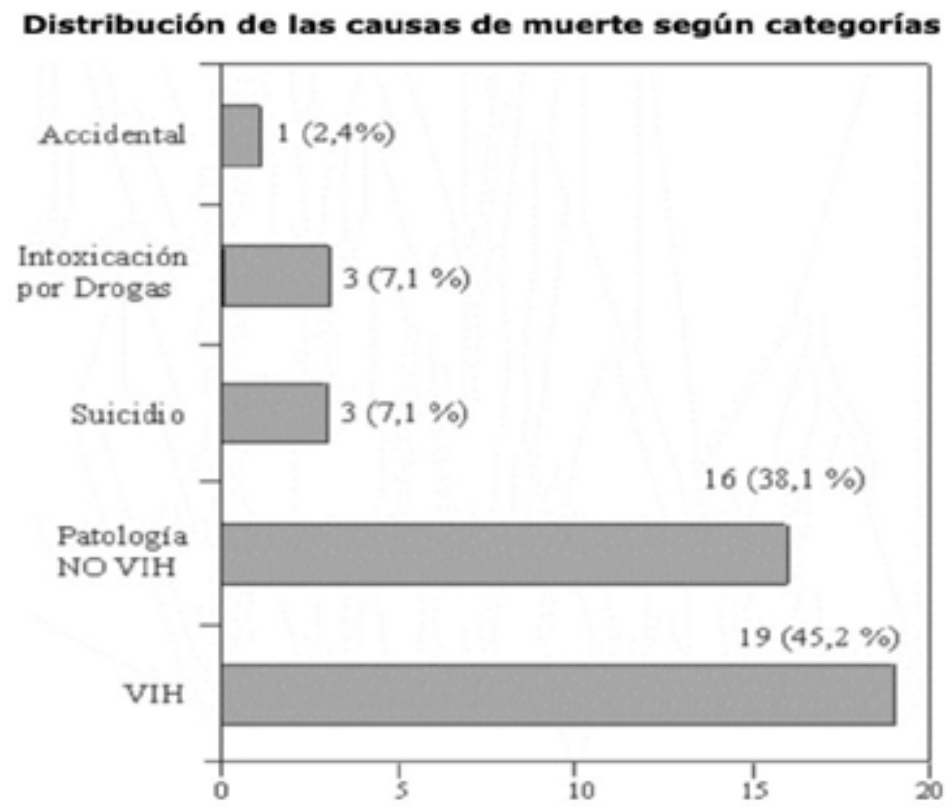

Fuente: Revista Española de Salud Pública. (Vera, E., Planelles, M \& García, J., 2005).

Además, instituciones supranacionales como la Organización Mundial del Comercio (OMC), junto con las empresas privadas, promulgan políticas que afectan a los países pobres. Por ejemplo, a principios de siglo, debido a la presión de los países en vías de desarrollo, la OMC emitió una declaración en la que se dictaba que los intereses de la salud pública debían tener prioridad sobre los reglamentos de la OMC, porque los precios de los medicamentos esenciales eran muy elevados; sin embargo, los países del hemisferio norte, influenciados por los intereses comerciales de las empresas farmacéuticas y sin ninguna intervención de la OMC, impusieron reglamentos alternativos para la protección de las patentes de los medicamentos. Esas reformas complicaron la producción de medicamentos genéricos asequibles, especialmente, en los países pobres. Por lo tanto, estos países siguen dependiendo de los costosos medicamentos patentados producidos en las naciones más ricas y los 
más afectados son las clases más pobres porque no pueden acceder a ese tipo de medicamentos (Mayne, 2002, p. 1-2). Esta situación se ha exacerbado durante la actual pandemia del Covid-19. James Zhan, director de inversiones y empresas de la Conferencia de las Naciones Unidas sobre Comercio y Desarrollo (UNCTAD, por sus siglas en inglés), sostiene que la demanda masiva para la vacuna contra el COVID-19 supera rápidamente la oferta y las poblaciones de los países pobres son las que se quedan sin acceso a estas ("COVID-19 heightens need", 2020). Esto ocurre como resultado de la falta de capacidad productiva en los países en vías de desarrollo. De los 40 fabricantes de vacunas de los 14 países que forman parte de la Red de Fabricantes de Vacunas de los Países en Desarrollo, solo tres están localizados en América Latina. El director ejecutivo de Biovac, Morena Makhoana, dijo que la seguridad de la salud pública de América Latina requiere una mayor producción local, de lo contrario, los 650 millones de personas de la región siguen siendo vulnerables a los choques en las cadenas de suministro mundial y las políticas comerciales de los gobiernos extranjeros ("COVID-19 heightens need", 2020).

Otro factor importante que contribuye al aumento de la pobreza son las decisiones políticas adoptadas por los países industrializados para promover conflictos armados en países en vías de desarrollo. Con el pretexto de promover libertad y democracia, los países occidentales se involucran en la política local de otras naciones. Esto suele dar lugar a procesos de violencia en los que las comunidades son desplazadas y prácticamente no tienen derechos (Balfour y Cadava, 2004, p. 282). En algunos casos, para proteger sus intereses económicos y políticos, los gobiernos extranjeros proporcionan armas, financiación y capacitación a los ejércitos locales. Por ejemplo, USA provee aproximadamente 3.000 millones de dólares al año al gobierno israelí para ser usados en su conflicto territorial con Palestina (Sparrow, 2014). Las guerras no solo aumentan la brecha de desigualdad entre los pobres y las clases pudientes, sino que, también, limitan el empleo y el acceso a la educación (Benatar, 1988 , p. 296). Por lo tanto, la pobreza aumenta considerablemente entre las comunidades más afectadas por los conflictos armados. 


\section{Conclusiones}

Aunque el derecho a la salud está protegido por las declaraciones y tratados de derechos humanos, la pobreza, el determinante social más importante de la salud, no se considera una violación de los derechos humanos. La pobreza es una consecuencia de la brecha de desigualdad entre las clases pudientes y el resto de la población y se hace más notoria en los países en vías de desarrollo. Algunas de las medidas económicas y políticas dictadas por las naciones ricas y las organizaciones supranacionales, contribuyen a agravar los niveles de desigualdad y pobreza. Por lo tanto, es necesario llevar a cabo una gran reforma que alivie la pobreza y disminuya la desigualdad, aunque vaya en contra del sistema económico mundial (Koubi, 2004, p. 330). Además, en lo que respecta al derecho a la salud, esta debería ser una preocupación no solo de las personas involucradas en las esferas de la salud, sino de todos los encargados de formular políticas y leyes para, de esta manera, desarrollar un sistema de salud pública igual para todos (Marmot, 2005 , p. 1099). Por último, los promotores de los derechos humanos como las Naciones Unidas deberían reconocer la gravedad que encierra la pobreza $y$, por consiguiente, deberían considerarla como una violación de los principios de derechos humanos. 


\section{Referencias bibliográficas}

Balfour, I \& Cadava, E. (2004). The Claims of Human Rights: An Introduction. The South Atlantic Quarterly, 103, 277-296. https://doi. org/10.1215/00382876-103-2-3-277

Benatar, S. (1998). Global Disparities in Health and Human Rights: A Critical Commentary. American Journal of Public Health, 88, 295-300. Doi: 10.2105/ajph.88.2.295

Bills, C \& Ahn, J. (2016). Global Health and Graduate Medical Education: A Systematic Review of the Literature. Journal of Graduate Medical Education, 8(5), 685-691. Doi: 10.4300/JGME-D-15-00774.1

Chen, J., Waterman, D., \& Krieger N. (2020). COVID-19 and the unequal surge in mortality rates in Massachusetts, by city/town and ZIP Code measures of poverty, household crowding, race/ethnicity, and racialized economic segregation. Harvard Center for Population and Development Studies, 19(2), 1-9. https://cdn1.sph.harvard.edu/wp-content/uploads/ sites/1266/2020/05/20_jtc_pdw_nk_COVID19_MA-excess-mortality_ text_tables_figures_final_0509_with-cover-1.pdf

Commission on Social Determinants of Health. (2008). Closing the gap in a generation: health equity through action on the social determinants of health. Geneva: World Health Organization Press. Doi: https://doi. org/10.1016/S0140-6736(08)61690-6

Constitution of the World Health Organization. (1967). World Health Organization, 1-20. https://apps.who.int/gb/bd/pdf_files/BD_49th-en.pdf

Coordinación General de Innovación en Métricas y Análisis de la Información. (2018). Reporte de pobreza y desigualdad. Instituto Nacional de Estadística y Censos, 1-9. https://www.ecuadorencifras.gob.ec/documentos/ web-inec/POBREZA/2018/Junio-2018/Informe_pobreza_y_desigualdad-junio_2018.pdf

Costa, F. (2008). Poverty and Human Rights: From Rethoric to Legal Obligations A critical Account of Conceptual Frameworks. International Journal on Human Rights, 5 (9), 80-107. https://sur.conectas.org/en/poverty-human-rights-rhetoric-legal-obligations/

COVID-19 heightens need for pharmaceutical production in poor countries. (2020). United Nations Conference on Trade and Development. https:// unctad.org/en/pages/newsdetails.aspx?OriginalVersionID $=2375$

FAQs: Global Poverty Line Update. (2015). The World Bank. http://www.worldbank.org/en/topic/poverty/brief/global-poverty-line-faq 
Gou, Z., Xie, X., Lu, Y \& Khoshbakht, M. (2018). Quality of Life (QoL) Survey in Hong Kong: Understanding the Importance of Housing Environment and Needs of Residents from Different Housing Sectors. International Journal of Environmental Research and Public Health, 15, 219. Doi:10.3390/ ijerph15020219

Katz, A. (2008). "New Global Health" A Reversal of Logic, History, and Principles. Social Medicine, 3, 1-3. http://www.socialmedicine.info/index.php/ socialmedicine/article/view/193/352

Koubi, G. (2004). Poverty as a human rights violation. International Social Science Journal, 56, 327-337. https://doi.org/10.1111/j.0020-8701.2004.00495.x

Marmot, S. (2005). Social Determinants of Health Inequalities. The Lancet, 365, 1099-1104. http://dx.doi.org/10.1016/S0140-6736(05)74234-3

Mayne, R. (2002). US Bullying on Drug Patents: One year after Doha. Oxfam International, 33, 1-17. http://policy-practice.oxfam.org.uk/publications/ us-bullying-on-drug-patents-one-year-after-doha-115060

Migration and inclusive societies. (s.f). United Nations Educational, Scientific and Cultural Organization (UNESCO). http://www.unesco.org/new/en/ social-and-human-sciences/themes/international-migration/glossary/ poverty/

Mikkonen, J \& Raphael, D. (2010). Social Determinants of Health: The Canadian Facts. Toronto: York University School of Health Policy and Management. https://thecanadianfacts.org/The_Canadian_Facts.pdf

Liu, Y., Zhang, Y., Liu, Z \& Wang, J. (2014). Gaps in studies of global health education: an empirical literature review. Global Health Action, 8(1), 1-9. https://doi.org/10.3402/gha.v8.25709

Llerena, F., Pinto, M, A., \& Pinto, M, C. (2017). Un poco de Protección Social: Seguros de Salud, Servicios de Salud y Pobreza. Foro Economía Ecuador. http://foroeconomiaecuador.com/fee/un-poco-de-proteccion-social-seguros-de-salud-servicios-de-salud-y-pobreza/

Pacto Internacional de Derechos Económicos, Sociales y Culturales. (1966). New York: United Nations. https://www.ohchr.org/sp/professionalinterest/ pages/cescr.aspx

Phillips, J. (2011). Thomas Pogge. World Poverty and Human Rights. Human Rights Review, 12, 405-407. https://doi.org/10.1007/s12142-011-0193-z

Pickett, K \& Wilkinson, R. (2015). Income inequality and health: A causal review. Social Science \& Medicine, 128, 317. https://doi.org/10.1016/j. socscimed.2014.12.031 
Poverty and social determinants. (s.f). World Health Organization. http://www. euro.who.int/en/health-topics/environment-and-health/urban-health/activities/poverty-and-social-determinants

Russell, G. (1998). All Rights Guaranteed-all Actors Accountable: Poverty is a Violation of Human Rights. Development in Practice, 8, 353-357. https:// doi.org/10.1080/09614529853657

Schuftan, C. (2012). Poverty and the Violation of Human Rights: A Proposed Conceptual Framework. International Journal of Health Services, 42, 485-498. https://doi.org/10.2190/HS.42.3.g

Social Determinants of Health: Know What Affects Health. (s.f). Center for Disease Control and Prevention. https://www.cdc.gov/socialdeterminants/

Social Determinants of Health: The Solid Facts. Second Edition (2003). Edited by Richard Wilkinson and Michael Marmot. Denmark: World Health Organization/Europe. http://www.euro.who.int/_data/assets/pdf_ file/0005/98438/e81384.pdf

Sparrow, T. (2014). Por qué EE.UU. le entrega millones en ayuda militar a Israel. BBC News. https://www.bbc.com/mundo/noticias/2014/08/140805_ eeuu_ayuda_militar_israel_tsb

The stark relationship between income inequality and crime. (2018). The Economist. https://www.economist.com/graphic-detail/2018/06/07/ the-stark-relationship-between-income-inequality-and-crime

United Nations. The Human Rights Based Approach to Development: Towards a Common Understanding Among UN Agencies. Inter-Agency workshop on Human Rights Based Approach in the context of UN Reform, Stamford, May 5-7 2003. https://unsdg.un.org/resources/human-rights-based-approach-development-cooperation-towards-common-understanding-among-un

Universal Declaration of Human Rights. (1948). Paris: United Nations. http:// www.un.org/en/udhrbook/pdf/udhr_booklet_en_web.pdf

Vera, E., Planelles, M \& García, J. (2005). Tendencia de la tasa de mortalidad en una prisión española. Revista Española de Salud Pública, 79, 673-682. http://scielo.isciii.es/scielo.php?script=sci_arttext\&pi$d=$ S1135-57272005000600007

What are human rights? (s.f). United Nations Human Rights Office of the High Commissioner. http://www.ohchr.org/EN/Issues/Pages/WhatareHumanRights.aspx 